\title{
COMPARISON OF FOUR LARYNGOSCOPES FOR OROTRACHEAL INTUBATION BY NURSES DURING RESUSCITATION WITH AND WITHOUT CHEST COMPRESSIONS: A RANDOMIZED CROSSOVER MANIKIN TRIAL
}

\author{
Iwona Stawicka ${ }^{1}$, Lukasz Czyzewski ${ }^{2}$, Jacek Smereka ${ }^{3}$, Lukasz Szarpak ${ }^{4}$ \\ ${ }^{1}$ Polish Society of Disaster Medicine, Poland \\ ${ }^{2}$ Department of Nephrologic Nursing, Medical University of Warsaw, Warsaw, Poland \\ ${ }^{3}$ Department of Emergency Medical Service, Wroclaw Medical University, Wroclaw, Poland \\ ${ }^{4}$ Department of Emergency Medicine, Medical University of Warsaw, Warsaw, Poland
}

\begin{abstract}
BACKGROUND: Currently, the gold standard for airway management in cardiopulmonary resuscitation is endotracheal intubation. This should be performed without interruptions in chest compressions, or with a short break only to introduce the tube.

METHODS: A total of 47 nurses were recruited who performed endotracheal intubation on a manikin in 2 scenarios: A - normal airway, without chest compressions; B - normal airway, with continuous chest compressions performed with the Lifeline ARM system. They used 4 devices: a Macintosh blade laryngoscope (MAC), and a TruView EVO2 (EVO2), TruView EVO2 PCD (PCD), and an ETView SL (ETView) laryngoscope. The intubation time and effectiveness, the grade of larynx visibility, and the ease of intubation in adults were compared.

RESULTS: The median time to first ventilation in scenario A was: for the MAC, 30.5 (interquartile range [IQR], 27-36.5); for the EVO2, 35.5 (IQR, 32-39.5): for the PCD, 26.5 (IQR, 25-28.5); and for the ETView, 23 (22-24.5)']; in scenario B: for the MAC, 47.5 (IQR, 37.5-51); for the EVO2, 42.5 (IQR, 39-47.5): for the PCD, 29.5 (IQR, 28-33); and for the ETView, 26 (IQR, 23-30.5) seconds]. The first inbunation attempt success rate in scrnario A was: for the MAC, 44.7\%; for the EVO2, 68.8\%; for the PCD, 82.9\%; and for the ETView, $91.5 \%$; in scenario B: for the MAC, $38.3 \%$; for the EVO2, 61.7\%; for the PCD, 70.2\%; and for the ETView $89.4 \%$.

CONCLUSIONS: The efficacy of endotracheal intubation by nurses turned out to be insufficient. Ongoing chest compressions significantly reduced the intubation effectiveness with the MAC. After a short training session, nurses could perform intubation with videolaryngoscopes. ETView appeared to be the most effective method in both scenarios.
\end{abstract}

KEY WORDS: cardiopulmonary resuscitation, endotracheal intubation

Disaster Emerg Med J 2016; (1)1: 14-23

\section{INTRODUCTION}

Sudden cardiac arrest (SCA) is among the main causes of death in Europe, affecting 350,000-
$-700,000$ persons per year, i.e. about $55-133$ cases per 100,000 inhabitants [1-3]. According to Gach et al., the incidence of SCA in the medical rescue 
teams operating in the Bielsko-Biala region in Poland is 117 cases per 100,000 inhabitants [4]. All these patients require immediate cardiopulmonary resuscitation (CPR), including advanced life support provided by medical personnel. Although first aid course participants (bystanders) are taught that in cases of their discomfort with mouth-to-mouth ventilation in an SCA patient they should perform chest compressions (CC) only [5], medical personnel should perform CPR with both CC and ventilation. It should be noted that the body oxygen reserve in SCA in normothermia is sufficient for about 3-5 min, after which time irreversible changes may develop in vital organs, particularly in the central nervous system, sensitive to hypoxia. Thus, clearing the airway, proper ventilation, and airway management are the key CPR elements. According to the current guidelines of the European Resuscitation Council (ERC) and the American Heart Association (AHA), the gold standard for airway management in CPR is endotracheal intubation [6, 7]. This consists of introducing an endotracheal tube to the larynx through the vocal cords, and inflating the air cuff to isolate the respiratory tract from the external environment, reducing the risk of gastric contents aspiration during potential regurgitation $[8,9]$.

The ERC and AHA guidelines also recommend that if the skills of the person performing endotracheal intubation allow it, there should be no interruptions in CC, or a short break only to introduce the tube through the vocal cords [6]. This is crucial because any CC interruption causes perfusion deterioration and reduces resuscitation effectiveness [10].

The aim of the study was to compare the intubation time and effectiveness, the grade of larynx visibility, as well as the ease of intubation in adults performed by nurses using 4 devices: a Macintosh blade laryngoscope (MAC), and a TruView EVO2 (EVO2), along with a TruView EVO2 PCD (PCD), and ETView SL (ETView) laryngoscope, in simulated CPR settings, with and without CC.

\section{METHODS}

This open prospective randomized crossover manikin study was approved by the Institute Review Board of the Polish Society of Disaster Medicine (approval No.: IRB/07/11/2015), and registered at the Clinical Trials register (www. clinicaltrials.gov, identifier NCT02804451).
With voluntarily written informed consent, 47 nurses were recruited who satisfied the following inclusion criteria, namely those who: (1) had not received any training in endotracheal intubation with videolaryngoscopy before the study; (2) had nursing specialization in anaesthesiology or emergency medicine; (3) presented no wrist or low back diseases, or pregnancy. All of them were routinely involved in SCA management and initial treatment in their professional work. The study was carried out between December 2015 and March 2016.

\section{Simulation scenarios}

Each participant performed endotracheal intubations on a SimMan 3G manikin (Laerdal, Stavanger, Norway) in 2 airway scenarios:

1. Scenario A: normal airway, without CC during intubation.

2. Scenario B: normal airway, with continuous $C C$ performed with the Lifeline ARM system (Defibtech, Guilford, USA) at a rate of $100 / \mathrm{min}$, to a depth of $4-5 \mathrm{~cm}$.

In each scenario, the manikin was placed on the floor in a neutral position in a bright room.

\section{Devices}

The study participants performed endotracheal intubation using 4 different devices (Figure 1):

1. A laryngoscope with a Macintosh blade size 3, as the 'gold standard' (MAC; Mercury Medical, Clearwater, USA).

2. A TruView EVO2 laryngoscope with blade size 3 (EVO2; Truphatek Holdings, Ltd., Netanya, Israel), applied as a type of optical laryngoscope.

3. A TruView EVO2 PCD laryngoscope with blade size 3 (PCD; Truphatek International Ltd., Netanya, Israel), used as a type of videolaryngoscope.

4. An ETView SL sized ID 7.0 (ETView; ETViewLtd, Misgav, Israel), used as an video endotracheal tube.

In all the intubation cases, an endotracheal tube size ID 7.0 (Covidien, Mansfield, USA) was applied and pre-moisturized with a lubricant. In the case of the MAC and ETView, a standard metal introducer was located in the tube light, whereas with the EVO2 and PCD, a special ready-made endotracheal tube guide was used. The participants also had a $10 \mathrm{ml}$ syringe at their disposal to inflate the tube cuff, and a self-inflating bag, utilized after introducing the endotracheal tube for a ventilation check and confirmation of a successful intubation. 

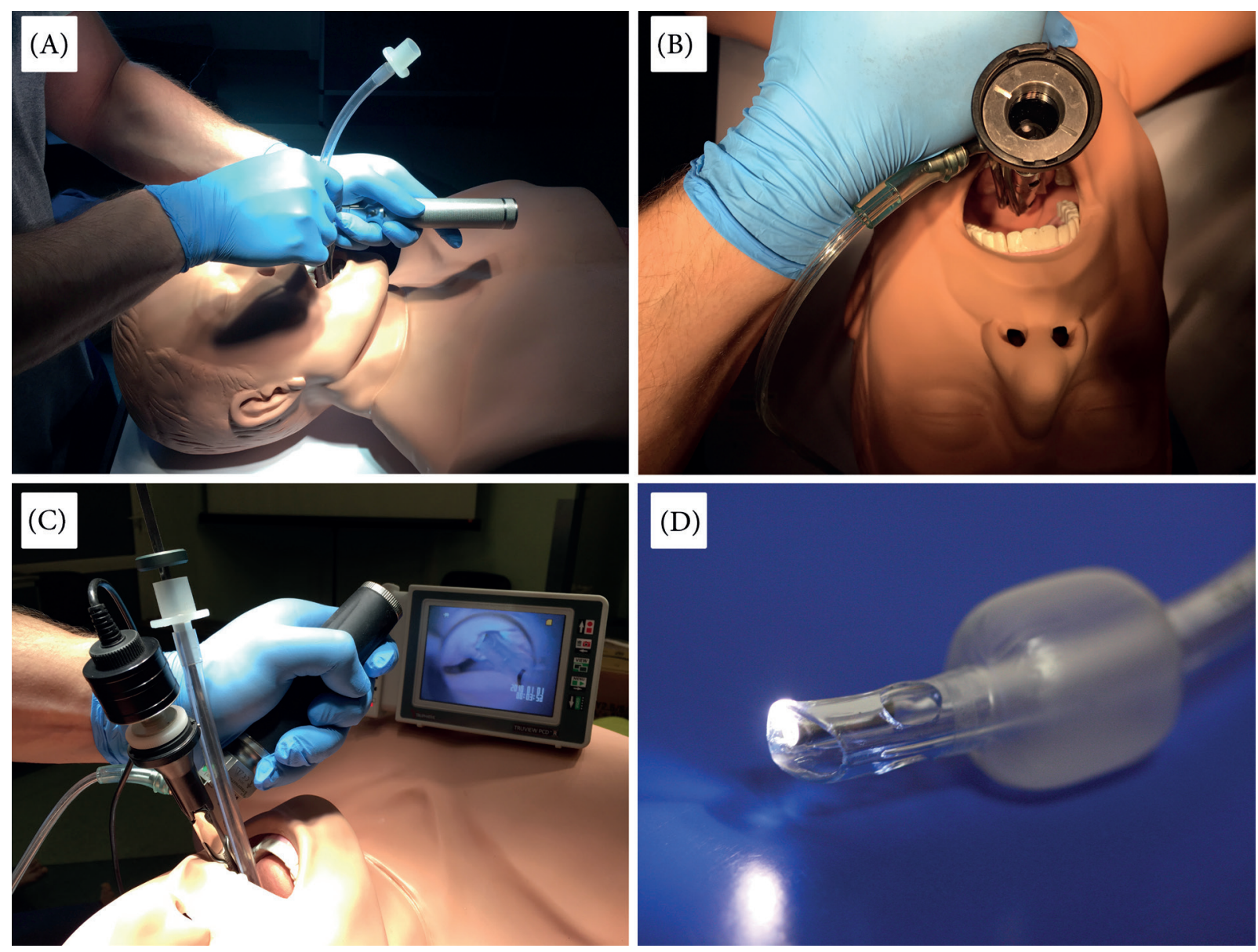

FIGURE 1. Laryngoscopes used in the study: (A) a Macintosh laryngoscope, (B) a TruView EVO2, (C) a TruView EVO2 PCD, (D) an ETView SL

\section{The study protocol}

Before the study, the participants were asked to complete a questionnaire verifying their knowledge of endotracheal intubation with the various assessed techniques. Although all of the nurses declared their ability to perform intubation using direct laryngoscopy, none of them had had experience in videolaryngoscopy. Then the participants received a 60 -min training session in the anatomy, physiology, and pathophysiology of breathing, which also referred to the various airway management techniques. The training session was conducted by a university lecturer experienced in endotracheal intubation. After the theoretical section and a demonstration of the correct endotracheal intubation techniques with the devices mentioned above, the participants received a practical training session. They performed endotracheal intubation using MAC, EVO2, PCD, and ETView on a SimMan $3 \mathrm{G}$ training manikin (Laerdal, Stavanger, Norway) in order to make sure they were familiar with the proper use of these devices. The session ended when each participant correctly performed 3 intubations each of the 4 devices.
Subsequently, with the use of the Research Randomizer software (www.randomizer.org), the participants were divided into 4 groups (Figure 2). The order of both the participants and the devices was random. The first group started intubation with the MAC in scenario $A$; the second, using the EVO2 in scenario $A$; the third, using the PCD in scenario $A$; the fourth, using the ETView in scenario A. After a maximum of 3 intubation attempts and a 20-min break, the nurses started endotracheal intubation using another method. In order to simulate the stress associated with emergency situations, the participants were informed that the SCA patients needed the quickest possible intubation.

\section{Outcomes}

The main measurement parameter was the time to the first ventilation attempt (T3), defined as the time from picking up the laryngoscope until the first ventilation attempt. In addition, the time to visualize the larynx (T1) and the time to introduce the endotracheal tube through the vocal cords (T2) were analysed (the participants informed the data collector when they saw the glottis opening). 


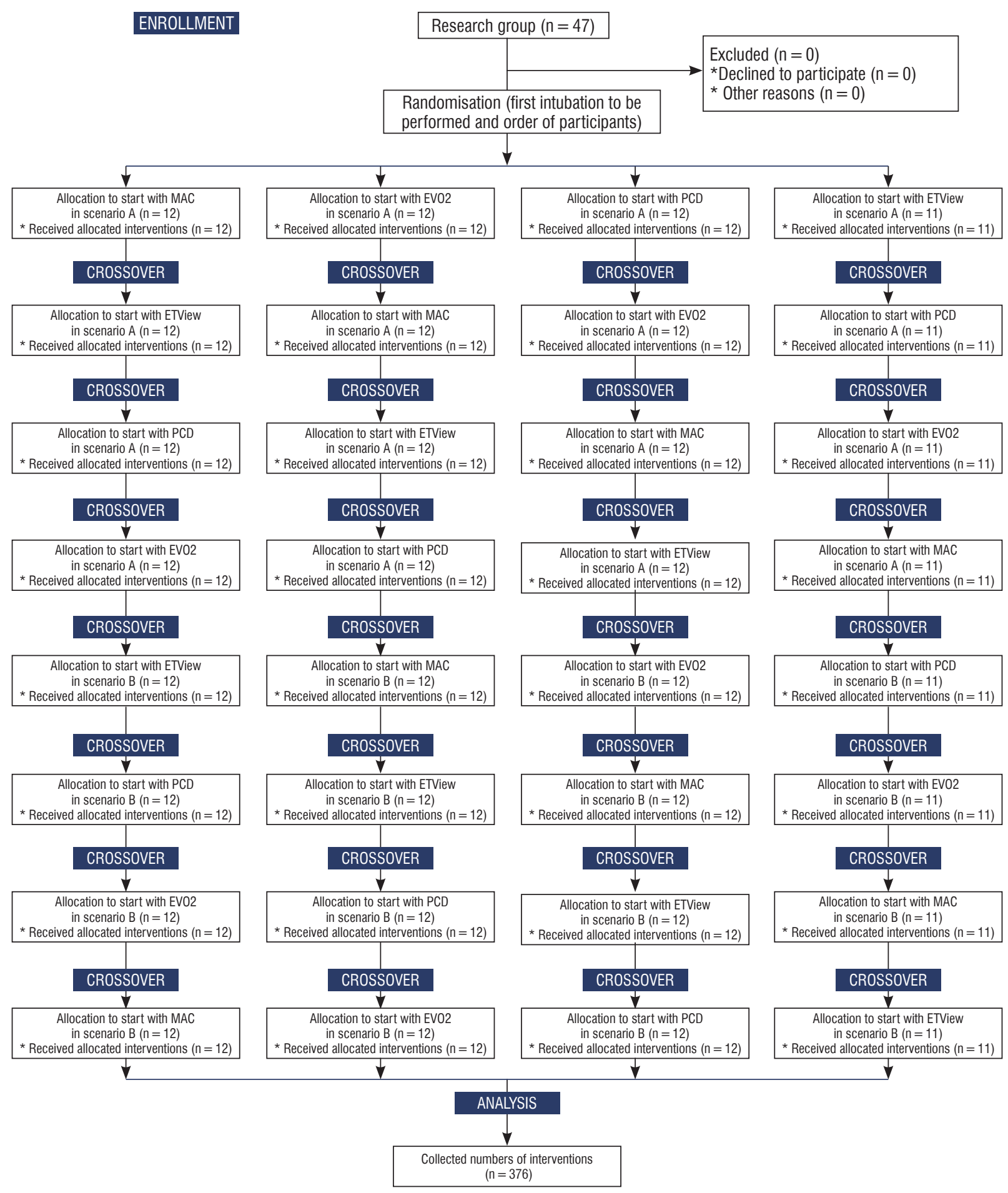

FIGURE 2. A study design and recruitment flow chart according to the CONSORT statement

Moreover, the effectiveness of the first intubation attempt and the overall intubation effectiveness were assessed. Intubation was considered effective when the tube passed the vocal cords and enabled efficient ventilation. After each intubation attempt, an investigator verified the tube position. The intubation attempt was deemed ineffective if the endotracheal tube was inserted into the oesophagus or the proce- dure lasted more than 120 s $[11,12]$. Furthermore, the degree of larynx visibility assessed by the participants was analysed based on the Cormack-Lehane scale [13]. Moreover, after the intubation attempts, the nurses were asked to assess the ease of each intubation technique. A 10-point visual analogue scale (VAS) was used, where 1 indicated an extremely simple procedure, while 10 indicated an extremely difficult one. 


\section{Sample size}

On the basis of pilot data, the assumptions of an alpha risk of 0.05 and a beta risk of 0.2 were made in order to calculate the number of participants to be included. The success rate of the first intubation attempt (during uninterrupted $C C$ ) in the pilot data when using the MAC, EVO2, PCD, and ETView varied and amounted to $30.5 \%, 63.3 \%, 72.4 \%$, and $92 \%$, respectively. The authors calculated that 29 participants would be required for the study (t-test, paired, two-sided). The participants were randomized with a 1:1 ratio.

\section{Statistical analysis}

Originally, the research material was collected in paper form, and then coded in Excel 2010 (Microsoft, Redmond, USA). A statistical analysis was performed with the Statistica EN 12.0 for Windows software (StatSoft, Tulsa, USA). The data were presented as mean and standard deviation (SD), median and interquartile ranges (IQR), as well as number and percentage (\%). The results were considered statistically significant at $p<0.05$. A normal distribution occurrence was confirmed by the Kolmogorov-Smirnov test. If the data did not present a normal distribution, non-parametric tests were used. In order to compare the intubation time between the groups, the Wilcoxon test for paired observations was applied. The McNemar test allowed one to evaluate differences in intubation effectiveness, while the Stuart-Maxwell test allowed one to compare the larynx visibility grade and ease of the procedure.

\section{RESULTS}

\section{Study participants}

The total of 47 nurses (39 female; $82.9 \%$ ) volunteered to participate in the study; $80.9 \%$ were specialized in anaesthesiologic nursing and $19.1 \%$ in emergency nursing. Their mean age was 34.5 (IQR, 26-37) years, while their mean work experience was 10.5 (IQR, 5-12.5) years.

\section{Scenario without chest compressions}

In this scenario, the time to first ventilation (T3) varied and amounted to $30.5,35.5,26.5$, and $23 \mathrm{~s}$ for the MAC, EVO2, PCD, and ETView, respectively (Figure 3).

A statistically significant difference was noticed between the MAC and EVO2 $(p<0.001)$, the PCD $(p<0.001)$, and the ETView $(p<0.001)$. There was

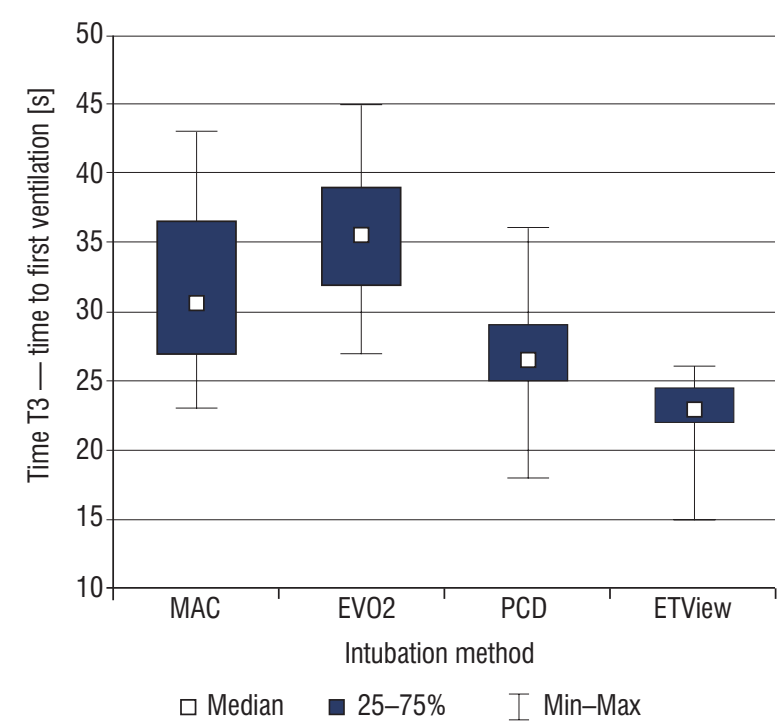

FIGURE 3. Comparison of the first ventilation time in the scenario without chest compressions

also a statistically significant difference in T3 between the EVO2 and PCD $(p<0.001)$ and the ETView $(p<0.001)$, as well as between the PCD and ETView $(p<0.001)$. The effectiveness of the first intubation attempt with the tested devices varied and amounted to $38.3 \%, 51.7 \%, 70.2 \%$, and $89.4 \%$ for the MAC, EVO2, $\mathrm{PCD}$, and ETView, respectively. Statistically significant differences in the efficacy of the first intubation attempt $(p<0.02)$ were observed when comparing MAC vs. EVO2, MAC vs. PCD, MAC vs. ETView, EVO2 vs. PCD, EVO2 vs. ETView, and PCD vs. ETView. The analysis of the overall intubation effectiveness (Table 1) showed a statistically significant difference between MAC and EVO2 $(p=0.015), P C D(p<0.001)$, and ETView $(p<0.001)$, between EVO2 and PCD $(p<0.001)$, and between EVO2 and ETView $(p<0.001)$.

According to the Cormack-Lehane scale, the best visualization of the larynx was observed in the case of the ETView, which was also considered the easiest and the most preferred intubation method.

\section{Scenario with chest compressions}

The parameters obtained during intubation with uninterrupted CC are presented in Table 2. There was a statistically significant difference in the time to the first ventilation attempt (T3, Figure 4$)$ between the MAC and EVO2 $(p=0.005)$, the PCD $(p<0.001)$, and the ETView $(p<0.001)$, between the EVO2 and PCD $(p<0.001)$ and the ETView $(p<0.001)$, and between the PCD and ETView $(p=0.013)$.

A statistically significant difference $(p<0.001)$ was noticed in the success of the first intubation 


\begin{tabular}{|l|c|c|c|c|}
\hline \multicolumn{1}{|c|}{ Table 1. Intubation data for resuscitation without chest compressions (scenario A) } \\
\hline \multicolumn{1}{|c|}{ Variable } & MAC & EVO2 & PCD & ETView \\
\hline Time to glottic visualization [s], median (IQR) & $12(10.5-14)$ & $12.5(10-15)$ & $10(8.5-12)$ & $8(6.5-11)$ \\
\hline Time to tube insertion [s], median (IQR) & $21(18-25.5)$ & $28(26.5-31)$ & $19(17.5-22)$ & $15(14-18.5)$ \\
\hline Time to first ventilation [s], median (IQR) & $30.5(27-36.5)$ & $35.5(32-39.5)$ & $26.5(25-28.5)$ & $23(22-24.5)$ \\
\hline First attempt success rate, n (\%) & $21(44.7 \%)$ & $32(68.8 \%)$ & $39(82.9 \%)$ & $43(91.5 \%)$ \\
\hline Overall success rate, n (\%) & $33(70.2 \%)$ & $41(87.2 \%)$ & $47(100 \%)$ & $47(100 \%)$ \\
\hline Cormack-Lehane score, n (\%) & $22(46.8 \%)$ & $34(72.3 \%)$ & $45(95.7 \%)$ & $47(100 \%)$ \\
1 & $25(53.2 \%)$ & $13(27.7 \%)$ & $2(4.3 \%)$ & - \\
2 & - & - & - & - \\
3 & - & - & - & - \\
\hline 4 & $4.5(3.8-5)$ & $5.3(4.5-5.5)$ & $3.3(3-3.8)$ & $2.6(1.7-2.9)$ \\
\hline VAS score (points), median (IQR) & $2(4.3 \%)$ & - & $13(27.6 \%)$ & $32(68.1 \%)$ \\
\hline Preferred airway device, n (\%) & & & & \\
\hline
\end{tabular}

IQR — interquartile range, VAS - visual analogue scale

attempt between the MAC and EVO2, the PCD, and the ETView; between the EVO2, PCD, and ETView; as well as between the PCD and ETView.

There was also a statistically significant difference in the overall success rate between the MAC and EVO2 $(p<0.001), \operatorname{PCD}(p<0.001)$, and ETView $(p<0.001)$; between the EVO2, PCD $(p=0.024)$, and ETView $(p<0.001)$; and between the PCD and ETView $(p<0.001)$.

The Cormack-Lehane score was statistically significant when the ETView was compared with the MAC $(p<0.001)$, EVO2 $(p<0.001)$, and PCD $(p<0.001)$. The preferred intubation method in this scenario was the ETView (in 44 of 47 participants).

\section{DISCUSSION}

The aim of the study was to evaluate the nurses' ability to perform endotracheal intubation in simulated CPR settings. A Macintosh laryngoscope as the 'gold standard' was used, as well as the EVO2, PCD and ETView. The nurses performed intubation in 2 scenarios during simulated CPR without CC (scenario A) and with ongoing uninterrupted CC (scenario B).

In scenario $A$, the efficacy of the first intubation attempt and the total efficacy using MAC equalled $44.7 \%$ and $70.2 \%$, respectively, with the median intubation time of $30.5 \mathrm{~s}$ (IQR, 27-36.5 s). Gaszyńska et al., in a group of 30 paramedics, noted that the necessary time to perform intubation with the MAC

\begin{tabular}{|l|c|c|c|c|}
\hline \multicolumn{1}{|c|}{ Table 2. Intubation data for resuscitation with chest compressions (scenario B) } \\
\hline \multicolumn{1}{|c|}{ Variable } & MAC & EVO2 & PCD & ETView \\
\hline Time to glottic visualization [s], median (IQR) & $15(13-21)$ & $15.5(12-19.5)$ & $14(11-16.5)$ & $10(7.5-13)$ \\
\hline Time to tube insertion [s], median (IQR) & $37(33-39.5)$ & $35(31-40.5)$ & $23(21.5-26)$ & $19(16-21.5)]$ \\
\hline Time to first ventilation [s], median (IQR) & $47.5(37.5-51)$ & $42.5(39-47.5)$ & $29.5(28-33)$ & $26(23-30.5)$ \\
\hline First attempt success rate, n (\%) & $18(38.3 \%)$ & $29(61.7 \%)$ & $33(70.2 \%)$ & $42(89.4 \%)$ \\
\hline Overall success rate, n (\%) & $25(53.2 \%)$ & $38(80.8 \%)$ & $41(87.2 \%)$ & $47(100 \%)$ \\
\hline Cormack-Lehane score, n (\%) & $15(31.9 \%)$ & $34(72.3 \%)$ & $44(93.6 \%)$ & $47(100 \%)$ \\
1 & $32(68.1 \%)$ & $13(27.7 \%)$ & $3(6.4 \%)$ & - \\
2 & - & - & - & - \\
3 & - & - & - & - \\
\hline 4 & $6.7(5-7.5)$ & $5.5(4.5-7)$ & $4(3-4.5)$ & $3.5(2.5-4)$ \\
\hline VAS score (points), median (IQR) & - & - & $3(6.4 \%)$ & $44(93.6 \%)$ \\
\hline Preferred airway device, $\mathrm{n}(\%)$ & & - & & \\
\hline
\end{tabular}

IQR — interquartile range, VAS — visual analogue scale 


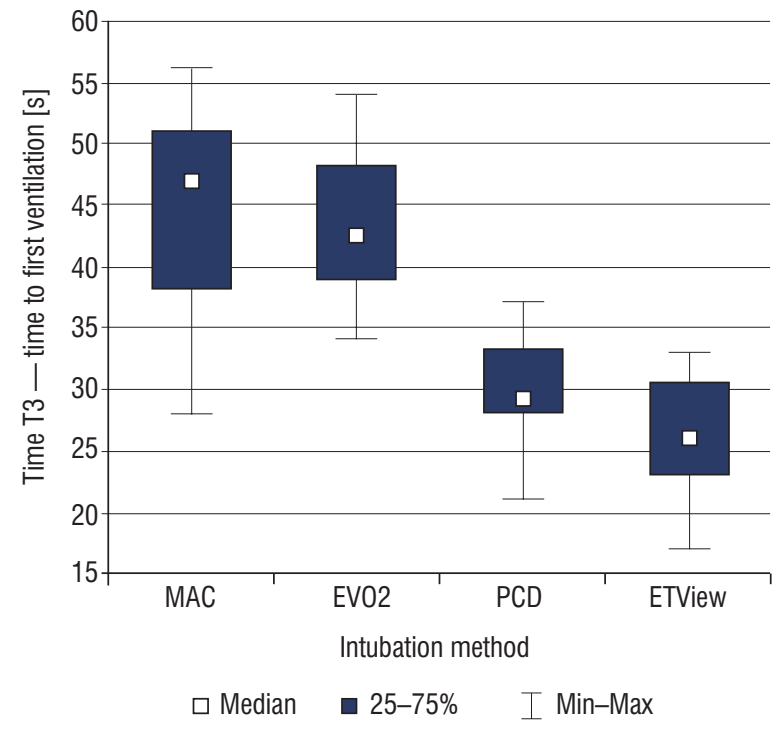

FIGURE 4. Comparison of the first ventilation time in the scenario with continuous chest compressions

was 17s (IQR, 13.23-22.29s), the efficacy of the first attempt was $73.3 \%$, and the total efficiency $100 \%$ [14]. The lower time needed for intubation and higher efficacy as compared with the present study, may be caused by paramedics' greater experience in direct laryngoscopy (working in rescue teams, they often face the necessity of endotracheal intubation). In the study by Gaszyńska et al., the paramedics' average professional experience amounted to $6.95 \pm 3.21$ years [14]. Han et al. observed a time required for MAC intubation of $18.10 \mathrm{~s}$ (IQR, 14.13-26.20s), with an overall intubation effectiveness of $93.8 \%$ [11]. The study by Han et al. involved 32 internship physicians, with limited experience in clinical endotracheal intubation. Similarly, in a study by Koyama, medical professionals inexperienced in endotracheal intubation were enrolled (11 physicians, 18 nurses, 4 medical students, and 2 paramedics), who were able to intubate in $22.7 \pm 11.2 \mathrm{~s}$ with an efficacy of $94.3 \%$ [15]. On the other hand, Kim indicated the efficacy of intubation performed by paramedics and paramedic students at a level of $90 \%$ in 18.33s (IQR, 14.08-21.44s) [16]. In a study conducted by Kurowski among paramedics, the mean MAC intubation time was $21.23 \pm 3.28 \mathrm{~s}$ [17].

In cases of intubation with EVO2, the nurses were able to achieve a first attempt effectiveness of $68.8 \%$ and an overall efficiency of $87.2 \%$ within 35.5 s (IQR, 27-36.5s). In the study by Gaszyńska et al., the efficacy of the first intubation attempt was $63.3 \%$, with a total efficiency of $90 \%$ and a median duration of 28.64s (24.02-38.34s) [14]. Singh showed an $88 \%$ effectiveness of EVO2 intubation performed in 28.6s [18]. Saxena et al., in a study among 140 surgical patients, observed that anaesthesiologists needed an average of $34.1 \mathrm{~s}$ to perform intubation using EVO2, which was a statistically significantly longer time than they required for direct laryngoscopy intubation (22.4s) [19]. Similarly, a longer intubation time using EVO2 as compared with MAC ( $33.06 \pm 5.6$ vs. $23.11 \pm 5.7 \mathrm{~s}$, respectively) was noted by Timanaykar et al. [20]. In a study by Malik et al., the overall efficacy of EVO2 intubation carried out by experienced anaesthesiologists in a simulation setting was $97.1 \%$, with a mean intubation time of 15s (IQR, 10-19s) [21]. A study by Szarpak et al., conducted in a simulated neonatal resuscitation setting, the efficacy of EVO2 intubation was $100 \%$, with an average procedure time of $25.3 \mathrm{~s}$ (IQR, 23-30.5s) [22].

In addition, after connecting a camera to the monitor, EVO2 can serve as a videolaryngoscope $(P C D)$, allowing one to perform endotracheal intubation. Nurses using PCD were able to intubate a patient in $26.5 \mathrm{~s}(\mathrm{IQR}, 25-28.5 \mathrm{~s})$; the effectiveness of the first intubation attempt was $92.9 \%$, and the total efficiency $100 \%$. The PCD intubation time was $21.10 \pm 5.64 \mathrm{~s}$ [23] in a study by Bag et al., and 52 (20-102) s in a study by Mutlak et al. [24].

Moreover, in a study by Tempe et al., the average time of PCD intubation in patients undergoing cardiac surgery was $60.47 \pm 27.45 \mathrm{~s}$ [25]. The same anaesthesiologists performed endotracheal intubation using direct laryngoscopy in $36.68 \pm 16.15 \mathrm{~s}$. In a study by Riveros, the PCD and MAC intubation time was $44 \mathrm{~s}(\mathrm{IQR}, 28-62 \mathrm{~s})$ and $23 \mathrm{~s}(\mathrm{IQR}, 21-28 \mathrm{~s})$, respectively [26]. Therefore, applying videolaryngoscopes by anaesthesiologists may extend the intubation time in patients with normal airways. This phenomenon has been observed in many studies, and is due to the fact that anaesthesiologists, routinely performing endotracheal intubation, are accustomed to standard laryngoscopes and find it difficult to switch to using video or optical laryngoscopes [26, 27]. An opposite trend is noticeable in representatives of specializations other than anaesthesiology or intensive care, as well as in other professionals, including paramedics or nurses [28].

The last device to be assessed was an endotracheal tube with a built-in camera connected to a monitor with a fibre optic cable, allowing one to receive a good quality image of the distal section of the endotracheal tube. The average time of intubation 
performed by nurses in this present study was $23 \mathrm{~s}$ (IQR, 22-24.5s), with a first attempt effectiveness of $91.5 \%$ and $100 \%$ total effectiveness. Thanks to the image from the end of the endotracheal tube, all the participants assessed the grade of larynx visibility as C/L1 on the Cormack-Lehane scale. Kurowski, in a group of physicians inexperienced in intubation, observed a first attempt effectiveness of $100 \%$ and an intubation time of 17s (IQR, 15-19s) [8]. Barak et al. assessed the ETView intubation time as $29 \pm 10$ s [29]. The higher intubation time probably resulted from difficulties in intubating obese patients.

Scenario B concerned intubation during CPR with uninterrupted CC. According to the current ERC guidelines, intubation - if the intubator's skills allow it - should be carried out without interruptions in CC. The mean intubation time with the MAC was $47.5 \mathrm{~s}(37.5-51 \mathrm{~s})$, while the first intubation attempt efficacy was $38.3 \%$, and the total efficiency $53.2 \%$. Gaszyńska et al. [14], Lee [30], among others, obtained lower values. Han et al. observed an intubation time with uninterrupted $C C$ at a level of 16.6s (IQR, 13.72-20.59s), and a total effectiveness of $81.3 \%$ [12]. In the study by Gaszyńska et al., the MAC intubation time was $16.6 \mathrm{~s}$, while the first attempt efficiency equalled $73.3 \%$ [14]. However, in another study Gaszyńska obtained a first attempt effectiveness of $53.3 \%$ and a total effectiveness of $80 \%$ [31]. In a study by Kim et al., paramedical students and paramedics intubated a manikin with ongoing CC and obtained a MAC intubation time of $24.14 \mathrm{~s}$ (IQR, 17.83-28.2s) with a effectiveness rate of $85 \%$ [16]. Similar results were noted by Kurowski et al.: 27 (IQR, 25-33s) [8]. However, Lee et al., analysing intubation performed by inexperienced intubators, observed a time of $35.0 \mathrm{~s}$ (IQR, 29.5-45.9s) and effectiveness of 78.9\% [30]. In a study by Szarpak et al., paramedics were able to intubate a manikin with MAC during simulated adult CPR with an efficiency rate of $88.9 \%$ in $18 \mathrm{~s}$ (IQR, 16-22s) [9], while in the case of child manikin intubation, they obtained $29.4 \pm 8.2$ s with $80.4 \%$ efficiency [9].

Regarding the EVO2, the endotracheal intubation time was $42.5 \mathrm{~s}(\mathrm{IQR}, 39-47.5 \mathrm{~s})$ which turned out to be statistically significantly longer than the MAC intubation time. In the studies by Gaszyńska, paramedics were able to perform intubation using MAC with uninterrupted CC within $36 \mathrm{~s}$ (IQR, 29-52s), with an effectiveness rate of the first intubation attempt of $73.3 \%$ and a total efficiency of $100 \%$ [14]; Szarpak et al., evaluating the intubation efficiency in newborns in CPR conditions, obtained an average intubation time of $25.3 \mathrm{~s}$ (23-30.5 s) and an overall effectiveness of $94.9 \%$ [22].

Among the study participants, $61.7 \%$ were able to perform endotracheal intubation using the $P C D$ in scenario $B$. The average time of intubation performed by nurses was $29.5 \mathrm{~s}$ (IQR, 28-33 s), and the overall efficiency $87.2 \%$. The study by Szarpak et al., $100 \%$ paramedics successfully performed PCD intubation in a mean time of 21.6s [32]. It is worth noting that the intubation in the study by Szarpak et al. was performed in a simulated child resuscitation setting and, according to numerous studies, the intubation efficacy in paediatric patients is lower than in adults.

In the present study, the efficacy of the first intubation attempt with the ETView was $89.4 \%$, while the overall efficacy equalled $100 \%$. The average time of intubation performed by the nurses was $26 \mathrm{~s}$ (23-30.5s). In the study by Kurowski et al., the time needed to perform intubation by physicians inexperienced in intubation was 19s (IQR, 15-22s), with a total efficiency of $100 \%$ [8].

As shown by scientific studies, CC during direct laryngoscopy significantly prolongs intubation $[8,22]$. When applying various videolaryngoscopy methods, as in the case the ETView, thanks to obtaining an image of the end of the laryngoscope blade or of the endotracheal tube, the differences in the efficiency between intubation with and without CC are not as significant as with the MAC [33-35].

The conducted study has some limitations. The first is that the endotracheal intubation was carried out on a manikin; however, only a manikin allows one to standardize study conditions. Moreover, according to the decision of the International Liaison Committee on Resuscitation, randomized crossover trials preformed during resuscitation are unethical [36]. Furthermore, applying multiple intubation attempts in a single patient would result in the risk of soft tissue damage or hypoxia. The second limitation is the fact that while only nurses participated in the study, this is an underappreciated group of medical staff who also face the need to apply airway control, including endotracheal intubation.

Beside these limitations, the study is also characterized by many strengths. Firstly, it was designed as a randomized crossover trial in order to avoid learning endotracheal intubation during the course of the study. Secondly, and advanced life support manikin was used for the intubation, and the most 
modern CC system, namely the Lifeline ARM, was utilized in order to standardize the difficulties arising from the CC. Another strength of the study is the employment of 4 different intubation techniques, including direct laryngoscopy, optical laryngoscopy, videolaryngoscopy, and an endotracheal videotube.

\section{CONCLUSIONS}

In the study group, the efficacy of endotracheal intubation performed by nurses was insufficient. Ongoing CC significantly reduces the effectiveness of MAC endotracheal intubation. After a short training session, nurses are able to perform highly efficient intubation with the use of videolaryngoscopes. Finally, the ETView turned out to be the most effective intubation method in both study scenarios.

Conflict of interest: None declared.

\section{REFERENCES}

1. Berdowski J, Berg RA, Tijssen JG, Koster RW. Global incidences of outof hospital cardiac arrest and survival rates: systematic review of 67 prospective studies. Resuscitation, 2010; 81: 1479-87.

2. Grasner JT, Bossaert L. Epidemiology and management of cardiac arrest: what registries are revealing. Best Pract Res Clin Anaesthesiol, 2013; 27: 293-306.

3. Grasner JT, Herlitz J, Koster RW, Rosell-Ortiz F, Stamatakis L, Bossaert L. Quality management in resuscitation — towards a European cardiac arrest registry (EuReCa). Resuscitation, 2011; 82: 989-94.

4. Gach D, Nowak JU, Krzych $\succeq$. Epidemiology of out-of-hospital cardiac arrest in Bielsko-Biała district: a 12-month analysis. Kardiol Pol, 2016 May 25. doi: 10.5603/KP.a2016.0086.

5. Perkins GD, Handley AJ, Koster RW et al. Adult basic life support and automated external defibrillation section Collaborators. European Resuscitation Council Guidelines for Resuscitation 2015: Section 2. Adult basic life support and automated external defibrillation. Resuscitation, 2015; 95: 81-99.

6. Soar J, Nolan JP, Böttiger BW et al. Adult advanced life support section Collaborators. European Resuscitation Council Guidelines for Resuscitation 2015: Section 3. Adult advanced life support. Resuscitation, 2015; 95: 100-147. doi: 10.1016/j.resuscitation.2015.07.016.

7. Link MS, Berkow LC, Kudenchuk PJ et al. Part 7: Adult Advanced Cardiovascular Life Support: 2015 American Heart Association Guidelines Update for Cardiopulmonary Resuscitation and Emergency Cardiovascular Care. Circulation, 132 (Suppl 2): S444-464. doi: 10.1161/ CIR.0000000000000261.

8. Kurowski A, Szarpak L, Truszewski Z, Czyzewski L. Can the ETView VivaSight SL Rival Conventional Intubation Using the Macintosh Laryngoscope During Adult Resuscitation by Novice Physicians?:
A Randomized Crossover Manikin Study. Medicine (Baltimore), 2015; 94(21): e850. doi: 10.1097/MD.0000000000000850.

9. Szarpak Ł, Czyżewski Ł, Truszewski Z, Kurowski A, Gaszyński T. Comparison of Coopdech ${ }^{\circledR}, \operatorname{CoPilot}{ }^{\circledR}$, Intubrite ${ }^{\circledR}$, and Macintosh laryngoscopes for tracheal intubation during pediatric cardiopulmonary resuscitation: a randomized, controlled crossover simulation trial. Eur J Pediatr, 2015;174(11): 1517-1523. doi: 10.1007/s00431-015-2567-8.

10. Koller AC, Salcido DD, Menegazzi JJ. Perishock Pause Intervals and Rearrest after Out-of-Hospital Cardiac Arrest. J Emerg Med, 2016; 50(2): 263-269.

11. Han SK, Shin DH, Choi PC. Utility of the Pentax-AWS without interruption of chest compression: comparison of the Macintosh laryngoscope with the Pentax-AWS in manikin model. Resuscitation, 2010; 81: 69-73.

12. Wetsch WA, Spelten 0 , Hellmich $M$ et al. Comparison of different video laryngoscopes for emergency intubation in a standardized airway manikin with immobilized cervical spine by experienced anaesthetists. A randomized, controlled crossover trial. Resuscitation, 2012; 83: 740-745.

13. Cormack RS, Lehane J. Difficult tracheal intubation in obstetrics. Anaesthesia, 1984; 39(11): 1105-1111.

14. Gaszynska E, Gaszynski T. Truview EVO2 and standard Macintosh laryngoscope for tracheal intubation during cardiopulmonary resuscitation: a comparative randomized crossover study. Medicine (Baltimore), 2014; 93(14): e78.

15. Koyama J, Iwashita T, Okamoto K. Comparison of three types of laryngoscope for tracheal intubation during rhythmic chest compressions: a manikin study. Resuscitation, 2010; 81(9): 1172-1174.

16. Kim YM, Kang HG, Kim JH, Chung HS, Yim HW, Jeong SH. Direct versus video laryngoscopic intubation by novice prehospital intubators with and without chest compressions: A pilot manikin study. Prehosp Emerg Care, 2011; 15(1): 98-103.

17. Kurowski A, Szarpak Ł, Zaśko P, Bogdański Ł, Truszewski Z. Comparison of direct intubation and Supraglottic Airway Laryngopharyngeal Tube (S.A.L.T.) for endotracheal intubation during cardiopulmonary resuscitation. Randomized manikin study. Anaesthesiol Intensive Ther, 2015; 47(3): 195-199.

18. Singh I, Khaund A, Gupta A. Evaluation of Truview evo2 Laryngoscope In Anticipated Difficult Intubation - A Comparison To Macintosh Laryngoscope. Indian J Anaesth, 2009; 53(2): 164-168.

19. Saxena A, Madan M, Shrivastava U et al. Role of the Truview EVO2 laryngoscope in the airway management of elective surgical patients: A comparison with the Macintosh laryngoscope. Indian J Anaesth, 2013; 57(3): 276-281.

20. Timanaykar RT, Anand LK, Palta S. A randomized controlled study to evaluate and compare Truview blade with Macintosh blade for laryngoscopy and intubation under general anesthesia. J Anaesthesiol Clin Pharmacol, 2011; 27(2): 199-204.

21. Malik MA, O'Donoghue C, Carney J, Maharaj CH, Harte BH, Laffey JG. Comparison of the Glidescope, the Pentax AWS, and the Truview EVO2 with the Macintosh laryngoscope in experienced anaesthetists: a manikin study. Br J Anaesth, 2009; 102(1): 128-134. 
22. Szarpak Ł, Kurowski A, Czyżewski $Ł$, Madziała M, Truszewski $Z$. Comparison of infant intubation through the TruView EVO2, TruView $P C D$, and Miller laryngoscope by paramedics during simulated infant cardiopulmonary resuscitation: A randomized crossover manikin study. Am J Emerg Med, 2015; 33(7): 872-875.

23. Bag SK, Kumar VR, Krishnaveni N, Ravishankar M, Velraj J, Aruloli M. A comparative study between Truview (PCD) laryngoscope and Macintosh laryngoscope in viewing glottic opening and ease of intubation: A crossover study. Anesth Essays Res, 2014; 8(3): 372-376.

24. Mutlak H, Rolle U, Rosskopf W, Schalk R, Zacharowski K, Meininger D, Byhahn C. Comparison of the TruView infant EV02 PCD ${ }^{\mathrm{TM}}$ and C-MAC video laryngoscopes with direct Macintosh laryngoscopy for routine tracheal intubation in infants with normal airways. Clinics (Sao Paulo), 2014; 69(1): 23-27.

25. Tempe DK, Chaudhary K, Diwakar A et al. Comparison of hemodynamic responses to laryngoscopy and intubation with Truview $\mathrm{PCD}^{\mathrm{TM}}$, McGrath $\AA$ and Macintosh laryngoscope in patients undergoing coronary artery bypass grafting: A randomized prospective study. Ann Card Anaesth, 2016; 19(1): 68-75.

26. Riveros R, Sung W, Sessler Dl et al. Comparison of the Truview PCD ${ }^{T M}$ and the GlideScope $(\circledR)$ video laryngoscopes with direct laryngoscopy in pediatric patients: a randomized trial. Can J Anaesth, 2013; 60(5): 450-457.

27. Yallapragada SV, Parasa M, Vemuri NN, Shaik MS. Comparison of the ease of tracheal intubation by postgraduate residents of anesthesiology using Airtraq ${ }^{\mathrm{TM}}$ and Macintosh laryngoscopes: An observational study. Anesth Essays Res, 2016; 10(2): 233-237.

28. Nouruzi-Sedeh P, Schumann M, Groeben H. Laryngoscopy via Macintosh blade versus GlideScope: success rate and time for endotracheal intubation in untrained medical personnel. Anesthesiology, 2009; 110(1): 32-37.
29. Barak M, Assalia A, Mahajna A, Bishara B, Braginski A, Kluger Y. The use of VivaSight ${ }^{\mathrm{TM}}$ single lumen endotracheal tube in morbidly obese patients undergoing laparoscopic sleeve gastrectomy. BMC Anesthesiol, 2014; 14: 31.

30. Lee DW, Kang MJ, Kim YH et al. Performance of intubation with 4 different airway devices by unskilled rescuers: manikin study. Am J Emerg Med, 2015; 33(5): 691-696.

31. Gaszynska E, Gaszynski T. Endotracheal intubation using the Macintosh laryngoscope or KingVision video laryngoscope during uninterrupted chest compression. Biomed Res Int, 2014; 2014: 250820. doi: 10.1155/2014/250820.

32. Szarpak Ł, Czyżewski Ł, Kurowski A, Truszewski Z. Comparison of the TruView PCD video laryngoscope and Macintosh laryngoscope for pediatric tracheal intubation by novice paramedics: a randomized crossover simulation trial. Eur J Pediatr, 2015; 174(10): 1325-1332.

33. Liu KP, Xue FS, Li CH. Videolaryngoscope for intubation during chest compression. J Anesth, 2015; 29(6): 975.

34. Szarpak Ł, Czyżewski Ł, Truszewski Z, Kurowski A. Pentax Airway Scope AWS-S200 video laryngoscope for child tracheal intubation in a manikin study with 3 airway scenarios. Am J Emerg Med, 2015; 33(9): 1171-1174.

35. Park SO, Kim JW, Na JH, Lee KH, Lee KR, Hong DY, Baek KJ. Video laryngoscopy improves the first-attempt success in endotracheal intubation during cardiopulmonary resuscitation among novice physicians. Resuscitation, 2015; 89: 188-194.

36. Bossaert LL, Perkins GD, Askitopoulou H, Raffay VI, Greif R, Haywood KL, Mentzelopoulos SD, Nolan JP, Van de Voorde P, Xanthos TT. Ethics of resuscitation and end-of-life decisions section Collaborators. European Resuscitation Council Guidelines for Resuscitation 2015: Section 11. The ethics of resuscitation and end-of-life decisions. Resuscitation, 2015; 95: 302-311. 